\title{
Induction of sleep apnoea with negative pressure ventilation in patients with chronic obstructive lung disease
}

\author{
Robert D Levy, Manuel G Cosio, Laurie Gibbons, Peter T Macklem, James G Martin
}

\begin{abstract}
Background Negative pressure ventilation provides intermittent non-invasive ventilatory assistance for patients with advanced chronic obstructive lung disease. Upper airway obstruction during sleep, a reported complication of the technique, may, however, limit its clinical applicability.

Methods The effects of nocturnal negative pressure ventilation on ventilation and on indices of sleep quality were investigated in five patients with severe chronic obstructive lung disease (mean (SE) FEV $131 \%$ (3\%) predicted) who had completed three months of nightly negative pressure ventilation. Subjects underwent overnight polysomnography on consecutive nights, the first night serving as a control and negative pressure ventilation being provided on the second night. Ventilators were adjusted to result in maximum suppression of the peak phasic electromyogram signal from the diaphragm.
\end{abstract}

Results Negative pressure ventilation resulted in substantial increases in episodes of obstructive apnoea and hypopnoea (mean (SE)/h 59.3 (19.8) v 3.2 $(1 \cdot 3)$ on control nights). Most obstructive events, however, were associated with under $3 \%$ oxygen desaturation, and the lowest recorded values for overnight oxygen saturation were similar on the two study nights. Negative pressure ventilation was also associated with significant increases in the frequencies of movement arousals and changes in sleep stage.

Conclusions Negative pressure ventilation applied during sleep to patients with advanced chronic obstructive lung disease may result in the development of recurrent episodes of apnoea and hypopnoea as well as altered sleep quality, which could limit its clinical applicability.

Negative pressure ventilation has been found to be effective for the provision of intermittent non-invasive assisted ventilation in patients with chronic hypercapnic respiratory failure resulting from neuromuscular disorders ${ }^{12}$ and thoracic deformities. ${ }^{34}$ Some recent studies have suggested that intermittent application of negative pressure ventilation may result in improvements in ventilatory performance in patients with severe chronic obstructive lung disease, ${ }^{5-7}$ possibly as a result of providing rest for chronically fatigued respiratory muscles.

Several investigators have reported upper airway obstruction to be a complication of negative pressure ventilation in both normal subjects ${ }^{8}$ and in patients with neuromuscular disorders. $^{9-13}$ To determine whether this phenomenon could limit the applicability of the technique, we examined the effects of negative pressure ventilation on breathing during sleep and sleep quality in patients with severe chronic obstructive lung disease.

\section{Methods}

SUBJECTS

We studied five subjects with severe, stable chronic obstructive lung disease. They were part of a large randomised, prospective clinical trial of ventilatory muscle rest. Details of the study design, protocols for selecting patients, and application of intermittent assisted ventilation have been reported elsewhere. ${ }^{14}$ The study protocol was approved by an ethics committee of the Montreal Chest Hospital Centre, McGill University, and informed consent was obtained from the subjects.

Subjects had just completed three months of intermittent assisted ventilation, during which they had been asked to apply the negative pressure ventilation for four to eight hours daily. They had been given the option of undergoing assisted ventilation during waking or sleep periods, or both. Most subjects in the larger trial of ventilatory muscle rest had chosen to use the equipment during the day, stating that use during sleep resulted in limitation of mobility, musculoskeletal discomfort, or a choking sensation. The five subjects enrolled in the present study were the first five to complete the trial who had chosen to use the equipment during sleep and agreed to participate in the study protocol.

\section{NEGATIVE PRESSURE VENTILATION}

Negative pressure ventilation was applied with a pressure cycled Thompson Maxivent ventilator (Thompson Respiration Products Inc, Boulder, Colorado) connected by a hose to an airtight pneumosuit (New Tech Medical Specialties, Palisades Park, New Jersey). Subjects were constrained by the equipment to remain in the supine or semi-recumbent position. 
To assess the activity of the inspiratory muscles, the electromyogram (EMG) of the diaphragm was recorded with surface electrodes placed over the right sixth and seventh intercostal spaces near the costal margin. ${ }^{15}$ The EMG signals were processed by a moving time averager (CWE Inc, Ardmore, Pennsylvania) with a time constant of $100 \mathrm{~ms}$ after band pass filtering (0.03-10 KHz) and rectification. Diaphragm EMG signals recorded from surface electrodes have been shown to provide a reasonable approximation to those recorded from a bipolar oesophageal electrode. ${ }^{16}$

The optimal ventilator settings-that is, those that resulted in the maximum reduction of spontaneous inspiratory efforts-were determined at the beginning of the study and were not changed subsequently. The ventilation frequency was set to equal the subject's spontaneous respiratory rate, and the inspiratory time: total time per breath ratio was fixed at 0.4 . The peak negative pressure in the pneumosuit was gradually increased until a maximum reduction in the phasic amplitude of the diaphragm EMG signal was observed.

\section{SLEEP STUDIES}

Subjects underwent two overnight polysomnographic studies on consecutive nights at the end of the three months of intermittent negative pressure ventilation. The first night served as a control night, during which subjects were fully instrumented and slept in the negative pressure ventilation set up while breathing spontaneously-although the ventilator cycled, it was not connected to the suit. On the second night negative pressure ventilation was applied with the ventilator settings determined at the beginning of the study.

Sleep was staged in 30 second epochs according to standard criteria. ${ }^{17}$ An episode of apnoea was defined as at least 10 seconds without airflow and of hypopnoea as 10 seconds with at least a $50 \%$ reduction in airflow as measured by thermocouples. Episodes of apnoea and hypopnoea had to be associated with movement arousals in order to be scored as such. Apnoea and hypopnoea episodes were divided into those associated with at least a 3\% decrement in oxygen saturation $\left(\mathrm{SaO}_{2}\right)$, and those with less than a 3\% reduction. On control nights obstructive apnoea was an episode in which respiratory effort was present, as indicated by thoracoabdominal movements measured by respiratory inductive plethysmography (Respitrace, AMI Inc, Ardsley, New York) as well as phasic EMG activity of the diaphragm. When no such effort was evident apnoea was considered to be of central origin. Mixed apnoea contained periods of both central and obstructive apnoea. As few episodes were observed on control nights, mixed and obstructive events were combined and called obstructive. With negative pressure ventilation apnoea was by definition obstructive as respiratory "effort" was provided by the ventilator.

ANALYSIS

The significance of differences in means between control and negative pressure ventilation
Table 1 Sleep characteristics on the two study nights for the five subjects (mean (SE) values)

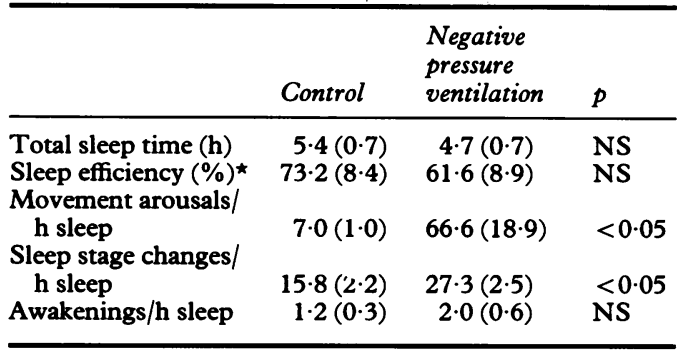

«Total sleep time/recording time $\times 100$.

nights was determined by Wilcoxon's signed rank test. ${ }^{18} \mathrm{p}$ Values below 0.05 were considered to be statistically significant. All data are reported as means with standard errors of the mean (SE) in parentheses.

\section{Results}

SUBJECTS' CHARACTERISTICS

Five subjects (three male, two female), aged 48-75 years, participated in the study. None was obese $(82-103 \%$ of predicted ideal body weight). The participants did not have histories suggesting sleep apnoea (excessive snoring, witnessed apnoea, daytime hypersomnolence). They had severe chronic obstructive lung disease with forced expiratory volume in one second $\left(\mathrm{FEV}_{1}\right) 31 \%$ (3\%) predicted, forced vital capacity (FVC) $48 \%(6 \%)$ predicted, arterial oxygen tension $\left(\mathrm{PaO}_{2}\right) 8.4(0.4) \mathrm{kPa}$, arterial carbon dioxide tension $\left(\mathrm{PaCO}_{2}\right) 5 \cdot 3(0 \cdot 4)$ $\mathrm{kPa}$, and $\mathrm{pH} 7 \cdot 4(0 \cdot 01))$.

All subjects had completed three months of daily intermittent negative pressure ventilation at home, and had chosen to use it during sleep. Internal clocks in the ventilators indicated a mean of $3.8(1.3)$ hours of use nightly.

\section{VENTILATORY INDICES}

The ventilator rates ranged from 16 to 24 breaths $/ \mathrm{min}$, and the peak negative pressures in the pneumosuits from -15 to $-30 \mathrm{~cm} \mathrm{H}_{2} \mathrm{O}$. These settings resulted in reductions in the

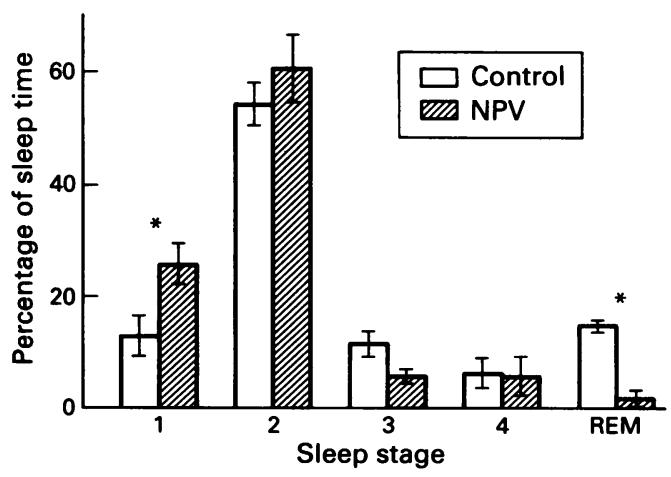

Sleep architecture on control and negative pressure ventilation (NPV) nights for the five study subjects (mean ( $S E$ ) values). Sleep with negative pressure ventilation was characterised by a decrease in the percentage of time spent in REM sleep and an increase in the percentage of stage 1 sleep $\left({ }^{\star} p<0.05\right)$. 
Table 2 Respiratory disturbances during sleep on the two study nights for the five subjects (mean (SE) values)

\begin{tabular}{|c|c|c|c|}
\hline & Control & $\begin{array}{l}\text { Negative pressure } \\
\text { ventilation }\end{array}$ & $p$ \\
\hline \multicolumn{4}{|l|}{$\begin{array}{l}\text { No of episodes of: } \\
\text { Apnoea } / \mathrm{h} \text { sleep }\end{array}$} \\
\hline Central & $1 \cdot 2(0 \cdot 6)$ & 一未 & \\
\hline \multicolumn{4}{|l|}{ Obstructive } \\
\hline $\begin{array}{l}<3 \% \text { desaturation } \\
\geqslant 3 \% \text { desaturation }\end{array}$ & $\begin{array}{l}0 \cdot 2(0 \cdot 1) \\
0.0(0 \cdot 0)\end{array}$ & $\begin{array}{l}4.3(1.9) \\
0.7(0.3)\end{array}$ & $\begin{array}{l}\text { NS } \\
\text { NS }\end{array}$ \\
\hline \multicolumn{4}{|l|}{ Hypopnoea/h sleep } \\
\hline $\begin{array}{l}<3 \% \text { desaturation } \\
\geqslant 3 \% \text { desaturation }\end{array}$ & $\begin{array}{l}1.4(0.9) \\
1.6(1.3)\end{array}$ & $\begin{array}{c}44 \cdot 6(21 \cdot 1) \\
9 \cdot 7(7 \cdot 3)\end{array}$ & $\begin{array}{l}<0.05 \\
\text { NS }\end{array}$ \\
\hline \multicolumn{4}{|l|}{ Obstructive apnoea and hypopnoea/h } \\
\hline$<3 \%$ desaturation & $1.6(1 \cdot 0)$ & $49 \cdot 0(20 \cdot 4)$ & $<0.05$ \\
\hline$\geqslant 3 \%$ desaturation & $1.6(1 \cdot 3)$ & $10 \cdot 4(7 \cdot 0)$ & $<0.05$ \\
\hline Total & $3 \cdot 2(1 \cdot 3)$ & $59 \cdot 3(19 \cdot 8)$ & $<0.05$ \\
\hline Apnoea or hypopnoea duration (s) & $18 \cdot 4(6 \cdot 0)$ & $16 \cdot 2(1 \cdot 2)$ & NS \\
\hline Baseline $\mathrm{SaO}_{2}(\%)$ (awake, supine) & $90 \cdot 1(2 \cdot 1)$ & $91 \cdot 2(1 \cdot 0)$ & NS \\
\hline Lowest overnight $\mathrm{SaO}_{2}(\%)$ & $81 \cdot 4(6 \cdot 0)$ & $85 \cdot 6(2 \cdot 3)$ & NS \\
\hline
\end{tabular}

^All episodes of apnoea with negative pressure ventilation were by definition obstructive (see text for details).

peak phasic amplitude of the diaphragm EMGs ranging from $64 \%$ to $92 \%$ of those recorded during spontaneous breathing when the subjects were awake.

\section{SLEEP STUDIES}

Subjects had more disrupted sleep on negative pressure ventilation nights than on control nights, as indicated by more movement arousals and changes in sleep stage (table 1). Furthermore, the sleep architecture (figure) was altered, with a reduction in rapid eye movement (REM) sleep from $15 \cdot 0 \%(1 \cdot 1 \%)$ of total sleep time on control nights to $2.0 \%$ $(1.5 \%)$ with negative pressure ventilation ( $\mathrm{p}<0.05)$, and an increase in stage 1 sleep $(54.2 \%(3.9 \%)$ v $60.7 \%(6.0 \%) ; \mathrm{p}<0.05)$. Whereas all subjects reached all sleep stages on control nights, only three had REM sleep with assisted ventilation. Despite the polysomnographic findings, three of the five subjects claimed to benefit from more restful sleep with negative pressure ventilation, and none had subjective complaints of excessive daytime somnolence after three months of nightly use.

Hypopnoea associated with a less than 3\% decrement in oxygen saturation was substantially more frequent on negative pressure ventilation nights than on control nights (table 2). Furthermore, although the overall mean (SE) frequency of apnoea and hypopnoea increased from $3 \cdot 2(1 \cdot 3)$ to $59 \cdot 3(19 \cdot 8)$ events hour $(p<0.05)$ on negative pressure ventilation nights, only a minority $(10 \cdot 4(7 \cdot 1))$ were associated with oxygen desaturation of $3 \%$ or more (table 2). The onset of obstructive apnoea and hypopnoea was associated with levels of inspiratory diaphragm EMG activity similar to those of the baseline before the onset of obstruction. The phasic activation increased with the progression of the apnoea or hypopnoea, reaching a maximum with the last obstructed breath.

On the control nights all episodes of obstructive apnoea and hypopnoea occurred during stages 1 and 2 and REM sleep. With negative pressure ventilation $6 \%$ of the events occurred during stage 3 or 4 . No relationship was evident for the group between the peak negative pressure applied and the apnoea-hypopnoea index.
Furthermore, there was no association between the degree of suppression of the phasic diaphragm EMG and the frequency of obstructive events.

The baseline oxygen saturation, recorded when subjects were awake and supine in the negative pressure ventilation set up, was similar on the two study nights (table 2). Despite the increased frequency of disordered breathing events on assisted ventilation nights, the lowest recorded overnight oxygen saturation was similar on control $(81.4 \%(6.0 \%))$ and negative pressure ventilation $(85.6 \%(2.3 \%))$ nights (table 2). The mean duration of episodes of apnoea and hypopnoea was also similar on control and negative pressure ventilation nights.

\section{Discussion}

Although the therapeutic role of ventilatory muscle rest in patients with chronic obstructive lung disease remains uncertain, preliminary data suggest that intermittent negative pressure ventilation may offer substantial benefit in such patients..$^{5-7}$ Upper airway obstruction has long been recognised to be a complication of negative pressure ventilation, and has been observed during sleep in normal subjects ${ }^{8}$ as well as in patients. ${ }^{9-13}$ The purpose of the present study was to define the extent of the problem in a group of patients for whom negative pressure ventilation was being evaluated as a means of providing intermittent ventilatory muscle assistance.

Our findings show that negative pressure ventilation during sleep results in the induction of intermittent upper airway obstruction as well as altered sleep characteristics in patients with severe chronic obstructive lung disease. In so far as very few patients in the larger trial of ventilatory muscle rest chose to sleep with negative pressure ventilation, the results for the sample studied with the current protocol are not likely to be representative of the findings in a randomly selected population. As our subjects decided to apply the apparatus during sleep, they may well have been those who were least affected in terms of upper airway obstruction.

The mechanism of the upper airway obstruc- 
tion observed with negative pressure ventilation has not been determined. As inspiration is generated by the ventilator, inspiratory muscle activation is suppressed. ${ }^{19}$ Obstruction is presumably the result of an absence, or reduction, of the phasic motor output of the respiratory centre to abductor and dilator muscles of the upper airway, which is required to counteract the transmural forces favouring collapse during inspiration. ${ }^{20}$ The importance of the normal temporal coordination of inspiratory activation of the upper airway and respiratory muscles for maintaining upper airway patency has previously been demonstrated in a patient undergoing diaphragm pacing. ${ }^{21}$ This line of reasoning is supported by a report by Goldstein and colleagues suggesting that the upper airway obstruction associated with negative pressure ventilation can be alleviated by the simultaneous application of nasal continuous positive airway pressure. ${ }^{22}$

Despite the dramatic increases in the frequency of disordered breathing events with negative pressure ventilation, this did not result in a decrease in the lowest overnight saturation. Furthermore, most episodes of apnoea and hypopnoea were associated with minimal oxygen desaturation. Presumably this was because the subjects studied had relatively intact arousal responses, as suggested by the fairly short duration of the events.

Of potential concern is the disruption of sleep in our subjects on the negative pressure ventilation night. Sleep quality is known to be poor in patients with chronic obstructive lung disease, being characterised by decreased total sleep time, reduced REM sleep, and frequent changes in sleep stage and arousals, possibly as a result of hypoxaemia or hypercapnia or both. ${ }^{23}$ Negative pressure ventilation resulted in a large increase in the frequency of movement arousals and a small but significant increase in the incidence of changes in sleep stage, as well as alteration of sleep architecture. The efficiency of sleep and frequency of awakenings were not affected. The subjects studied did not complain of increased sleep disturbance or excessive daytime somnolence after three months of nightly negative pressure ventilation, which brings into question the clinical relevance of the measured sleep disturbance. This is not, however, completely surprising because the frequency of apnoea and hypopnoea and indices of sleep fragmentation are not strongly correlated with excessive daytime somnolence in patients with obstructive sleep apnoea. $^{2425}$

The results of this study indicate that the application of nocturnal negative pressure ventilation in patients with chronic obstructive lung disease may result in the development of recurrent episodes of apnoea and hypopnoea as well as disrupted sleep. Consideration should be given to the performance of polysomnography in patients for whom negative pressure ventilation is prescribed.
1 Curran FJ. Night ventilation by body ventilators for patients in chronic respiratory failure due to late stage Duchenne muscular dystrophy. Arch Phys Med Rehabil 1981; 62:270-4.

2 Garay SM, Turino GM, Goldring RM. Sustained reversal of chronic hypercapnia in patients with alveolar hypoventilation syndromes: long term maintenance with noninvasive nocturnal mechanical ventilation. $A m \mathrm{~J}$ Med 1981;70:269-74.

3 Wiers PWJ, LeCoultre R, Dallinga OT, Van Dijl W, Meinesz AF, Sluiter HJ. Cuirass respirator treatment of chronic respiratory failure in scoliotic patients. Thorax 1977;32:221-8.

4 Kinnear W, Hockley S, Harvey J, Shneerson J. The effects of one year of nocturnal cuirass-assisted ventilation in chest wall disease. Eur Respir J 1988;1:204-8.

5 Braun NMT, Marino WD. Effect of daily intermittent rest of respiratory muscles in patients with severe chronic airflow limitation. Chest 1984;85:59-60S.

6 Cropp AJ, DiMarco AF. Effects of intermittent negative pressure ventilation on respiratory muscle function in patients with severe chronic obstructive pulmonary disease. Am Rev Respir Dis 1987;135:1056-61.

7 Guttierez M, Beroiza T, Contreras G, Diaz O, Cruz E, Moreno $R$, et al. Weekly cuirass ventilation improves blood gases and inspiratory muscle strength in patients with chronic airflow limitation and hypercarbia. Am Rev Respir Dis 1988;138:617-23.

8 Levy RD, Bradley TD, Newman SL, Macklem PT, Martin JG. Negative pressure ventilation: effects on ventilation during sleep in normal subjects. Chest 1989;95:95-9.

9 Elam JO, Hemingway A, Gullickson G, Visscher MB. Impairment of pulmonary function in poliomyelitis. Arch Intern Med 1948;81:649-65.

10 Peltier LF. Obstructive apnea in artificially hyperventilated subjects during sleep. J Appl Physiol 1953;5:614-8.

11 Scharf SM, Feldman NT, Goldman MD, Haut HZ, Bruce E, Ingram RH. Vocal cord closure: A cause of upper airway obstruction during controlled ventilation. $A m$ Rev Respir Dis 1978;117:391-7.

12 Ellis ER, Bye PTP, Bruderer JW, Sullivan CE. Treatment of respiratory failure during sleep in patients with neuromuscular disease: positive pressure ventilation through a nose mask. Am Rev Respir Dis 1987;135:148-52.

13 Bach JR, Penek J. Obstructive sleep apnea complicating negative-pressure ventilatory support in patients with chronic paralytic/restrictive ventilatory dysfunction. Chest 1991;99:1386-93.

14 Shapiro SH, Macklem PT, Gray-Donald K, Martin JG, Ernst PP, Wood-Dauphinee S, et al. A randomized clinical trial of negative pressure ventilation in severe chronic obstructive pulmonary disease: design and methods. J Clin Epidemiol 1991;44:483-96.

15 Gross D, Grassino A, Ross WRD, Macklem PT. Electromyogram pattern of diaphragmatic fatigue. $J$ Appl Physiol 1979;46:1-7.

16 Carrey Z, Gottfried SB, Levy RD. Ventilatory muscle support in respiratory failure with nasal positive pressure ventilation. Chest 1990;97:150-8.

17 Rechtschaffen A, Kales A. A manual of standardized terminology, techniques and scoring system for sleep stages of human subjecis. Washington, DC: US Government Printing Office, 1968. (National Institutes of Health publication No 204.)

18 Colton T. Statistics in medicine. Boston: Little, Brown and Company, 1974:219-21.

19 Levy RD, Carrey Z, Macklem PT, Martin JG. Suppression of ventilatory muscle activity in COPD patients with negative pressure ventilation [abstract]. Am Rev Respir Dis 1988;137:151.

20 Issa FG, Sullivan CE. Upper airway closing pressures in obstructive sleep apnea. J Appl Physiol 1984;57:520-7.

21 Hyland RH, Hutcheon MA, Perl A, Bowes G, Anthonisen NR, Zamel N, et al. Upper airway occlusion induced by diaphragm pacing for primary alveolar hypoventilation: implications for the pathogenesis of obstructive sleep apnea. Am Rev Respir Dis 1981;124:180-5.

22 Goldstein RS, Molotiu N, Skrastins R, Contreras M, Phillipson EA. Negative pressure ventilation in respiratory failure [abstract]. Am Rev Respir Dis 1986; 133:A167.

23 Fleetham J, West P, Mezon B, Conway W, Roth T, Kryger $M$. Sleep, arousals and oxygen desaturation in chronic obstructive pulmonary disease. The effect of oxygen therapy. Am Rev Respir Dis 1982;126:429-33.

24 Guilleminault C, Partinen M, Quera-Salva MA, Hayes B, Dement WC, Nino-Murcia G. Determinants of daytime sleepiness in obstructive sleep apnea. Chest 1988;94:32-7.

25 Roehrs T, Zorick F, Wittig R, Conway W, Roth T. Predictors of objective level of daytime sleepiness in patients with sleep-related breathing disorders. Chest 1989;95:1202-6. 